\title{
Aqueous Extracts of Mushrooms Enriched with Ergothionein with Antioxidant Activity and Its Effect on Human Dermal Fibroblasts UV irradi- ated
}

I International Congress of Biotechnology, Environment, Chemistry and Food

Corresponding Author:

Anabell Del Rocío

Urbina-Salazar

gata-any@hotmail.com

Published: 29 August 2021

Production and Hosting by Knowledge E

(c) Anabell Del Rocío Urbina-Salazar et al. This article is distributed under the terms of the Creative Commons Attribution License, which permits unrestricted use and redistribution provided that the original author and source are credited.

\section{Extractos Acuosos de Hongos Enriquecidos con Ergotioneina con Actividad Antioxidante y Su Efecto en Fibroblastos Dérmicos Humanos Irradiados con UV}

Anabell Del Rocío Urbina-Salazar ${ }^{1,2}$, Alberto Renato Inca-Torres ${ }^{1,3}$, Bryan Anthony Urbina-Salazar ${ }^{3}$, Valeria Fernanda Inca-Torres ${ }^{4}$, and Juan Bautista ${ }^{2}$

1'Universidad Técnica de Manabí, Instituto de Ciencias Básicas, Portoviejo, Ecuador

${ }^{2}$ Universidad de Sevilla, Facultad de Farmacia, Departamento de Bioquímica y Biología Molecular, Sevilla, España

${ }^{3}$ Universidad Técnica de Ambato, Facultad de Ciencias de la Salud, Carrera de Medicina, Ambato, Ecuador

${ }^{4}$ Hospital Básico Baños, Baños, Ecuador

\section{Abstract}

Ergotioneine (ERG) has potential applications as food additive and as an antioxidant for the treatment and/or prevention of diseases related to oxidative stress. In this work, we study the nature of antioxidant activity and investigate the effect of aqueous extracts of Agaricus bisporus enriched with ergotioneine (EAHE-ERG), a natural product, obtained through enzymatic digestion and membrane technology, in cellular response induced by UV, in crops fibroblasts. EAHE-ERG suppressed by the increase of TNF- $\alpha$ by UV-B radiation. Furthermore, in fibroblasts exposed to UV-A, EAHE-ERG suppressed the expression of matrix metalloproteinase-1 protein (MMP-1) in almost 50\% and reduced the RNAm expression from MMP-1. From these results, we conclude that EAHE-ERG can reduce anti-aging effects of the skin from UV radiation by removing $\mathrm{O}_{2}$ and $1 \mathrm{O}_{2}$, and the reduction protease signs and inflammatory activity.

Keywords: Ergotioneine, Agaricus bisporus, Antioxidant, Nutraceutical, Anti-inflammatory.

\section{Resumen}

La ergotioneina (ERG) tiene aplicaciones potenciales como un aditivo alimentario y como un antioxidante para el tratamiento y/o prevención de enfermedades relacionadas con el estrés oxidativo. En este trabajo estudiamos la naturaleza de la actividad antioxidante e investigamos el efecto de los extractos acuosos de Agaricus bisporus enriquecidos con ergotioneina (EAHEERG), un producto natural, obtenido mediante digestión enzimática y tecnología de membrana, en respuesta celular inducida por UV, en fibroblastos cultivados. EAHE-ERG suprimió la regulación por incremento de TNF- $\alpha$ mediante radiación UV-B. Además, en fibroblastos expuestos a UV-A, EAHE-ERG suprimió la expresión de proteína de metaloproteinasa-1 de matriz (MMP-1) en casi 50\% y redujo la expresión de ARNm de MMP-1. A partir de estos 
resultados, concluimos que EAHE-ERG puede reducir los efectos antienvejecimiento de la piel después de la irradiación UV mediante la eliminación de $\mathrm{O}_{2}$ y $1 \mathrm{O}_{2}$, y la reducción de las señales de proteasa y actividad inflamatoria.

Palabras Clave: Ergotioneina, Agaricus bisporus, Antioxidante, Nutracéutico, Antiinflamatorio.

\section{Introducción}

La ergotioneina (ERG), es un derivado de tiourea de histidina con potentes actividades antioxidantes, es producido por pocos organismos como actinobacterias, cianobacterias y ciertos hongos, entre otros, pero no es sintetizado por plantas y animales. Se ha demostrado que los eucariotas superiores contienen ERG, pero hasta la fecha no se ha informado que estos la biosintetizan [1, 2], se cree que las plantas toman la ERG excretada por los hongos del suelo mediante relaciones simbióticas [3, 4]. La ERG se produce casi exclusivamente a partir de la dieta en humanos, se acumula en varias partes del cuerpo humano como la médula ósea, el hígado, los eritrocitos, los riñones, los líquidos seminales, los ojos y la piel [5], es transportado mediante un transportador específico SLC22A4, la selección natural de dicho transportador implica que la ERG está involucrada en el mantenimiento de la salud o la mitigación de la enfermedad, acumulándose en los sitios de lesión [6,7].

La ERG exhibe aplicaciones potenciales debido a las propiedades antioxidantes que posee como: Eliminación de radicales libres y especies reactivas de oxígeno [8] y la quelación de iones metálicos divalentes, en el tratamiento o prevención de enfermedades relacionadas con el estrés oxidativo, además que protege a las células del ADN mitocondrial asociado al fotoenvejecimiento [4, 9], debido a estos efectos beneficiosos podría representar un alto valor en el mercado mundial de suplementos dietéticos [6].

El fotoenvejecimiento de la piel está relacionado con una combinación de factores intrínsecos y extrínsecos que podrían cambiar los aspectos funcionales y estructurales de la piel, causado por cambios en las expresiones génicas en los fibroblastos dérmicos [10]. Además, ha demostrado posibles efectos in vivo de varias enfermedades neurodegenerativas [11], en la lesión por isquemia reperfusión [12], entre otras.

Actualmente, la ERG comercial se sintetiza químicamente, siendo una alternativa sostenible de bajo costo la producción en fábricas de células microbianas [13, 14]. En un estudio anterior se cuantificó ERG en Agaricus bisporus identificando que contienen del 0,20-0,31 mg/g, [15, 16], además se evaluó el efecto en extractos naturales de A. bisporus observándose que tienen la capacidad de reducir significativamente el contenido de lípidos intracelulares, el tamaño de las gotas de lípidos y el contenido de TG intracelular [17]. En este estudio presentamos un producto natural enriquecido en ERG natural en forma de extracto, EAHE-ERG, obtenido de $A$. bisporus, estudiamos la naturaleza de la actividad antioxidante e investigamos su efecto en respuesta celular inducida por UV. 


\section{Materiales y Métodos}

\subsection{Preparación de EAHE-ERG}

Se utilizó como materia prima A. bisporus, que se obtuvo del Grupo Riberebro (La Rioja, España). EAHE-ERG se obtuvo siguiendo el procedimiento descrito por Cremades et al., usando enzimas y tecnología de membrana [15]. La Figura 1 muestra esquemáticamente el procedimiento.

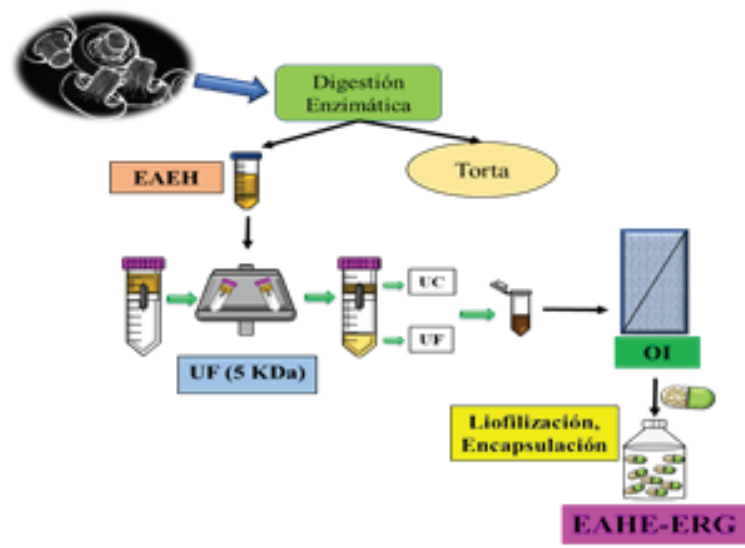

Figure 1

Procedimiento para la extracción de EAHE-ERG.

\subsection{Cultivos celulares}

Los fibroblastos humanos primarios se cultivaron en medio DMEM estándar (Invitrogen, Carlsbad, CA, EE. UU.), Suplementado con 10\% de suero bovino fetal (Invitrogen, Carlsbad, CA, EE. UU.), $10 \mathrm{mM}$ HEPES, $2 \mathrm{mM}$ L-glutamina y antibióticos (100 U/mL de penicilina, $0,25 \mu \mathrm{g} / \mathrm{mL}$ de sulfato de estreptomicina) y $50 \mu \mathrm{g} / \mathrm{mL}$ de uridina, con o sin L-ergotioneina $20 \mu \mathrm{M}$ (EAHE-ERG o ERG pura) a $37^{\circ} \mathrm{C}$ en $5 \%$ de $\mathrm{CO}_{2}$.

\subsection{Irradiación UVB}

Las células confluentes (control y tratadas con EAHE-ERG o ERG pura [L-ergotioneina $20 \mu \mathrm{M}]$ ) se expusieron a UVB (300 J/m², bombilla fluorescente TL12 Philips, pico de emisión a $313 \mathrm{~nm}$ ) a través de solución salina tamponada con fosfato.

\subsection{Medición de la concentración de ROS intracelular}

La concentración de ROS se midió utilizando el indicador general de estrés oxidativo $\mathrm{CM}-\mathrm{H}_{2}$ DCFDA (derivado de clorometilo de $\mathrm{H}_{2}$ DCFDA). 


\subsection{Determinación de Ergotioneina por HPLC}

La concentración de ERG se determinó mediante HPLC, siguiendo el método de Dubost et al. [18]. Como estándar se usó la L-ergotioneina sintética (OXIS International, Japón).

\subsection{Ensayo TNF- $\alpha$}

El TNF- $\alpha$ se cuantificó utilizando kits comerciales de ELISA (BD Biosciences, San Diego, CA, EE. UU.)

\subsection{Ensayo MMP-1}

La expresión de la metaloproteinasa-1 de la matriz (MMP-1) se evaluó mediante análisis de inmunotransferencia (Western Blot) y la actividad se ensayó con un kit de ensayo de actividad MMP-1 (Enzo Life Sciences, Farmingdale, NY, EE. UU.).

\subsection{Análisis estadístico}

Los datos se expresan como medias \pm DE (Desviación Estándar) y se analizaron mediante ANOVA unidireccional seguido de la prueba LSD protegida de Fisher para múltiples comparaciones de medias grupales; se considera un valor de $p<0,05$ estadísticamente significativo.

\section{Resultados}

Se utilizó $A$. bisporus como materia prima, se cuantificó ERG con una concentración de $0,21 \pm 0,03 \mathrm{mg} / \mathrm{g}$ de ERG/g de peso seco, resultados similares a los obtenidos por Cremades et al. $(2015)[15,16]$ pero en concentraciones más bajas que las encontradas en otros hongos [18]. Sin embargo al ser una materia prima que se puede obtener en grandes cantidades representa una fuente importante de ERG con un alto potencial antioxidante. Los resultados de la concentración de ERG se muestran en la Tabla 1, cómo se puede observar la cantidad de ERG varía en cada proceso, obteniéndose EAHE-ERG con una concentración de $175,01 \mathrm{mg}$, y con un porcentaje de rendimiento del 93,52\%, con respecto al producto inicial, cantidad muy significante de ERG.

\subsection{Efecto protector contra el estrés oxidativo celular}

La concentración intracelular de ROS (reactive oxygen species) se midió utilizando el indicador general de estrés oxidativo $\mathrm{CM}-\mathrm{H}_{2}$ DCFDA. Los resultados obtenidos se muestran en la Figura 2, observándose que EAHE-ERG presenta un alto porcentaje de antioxidante al igual que un extracto con ERG pura, lo que indica que EAHE-ERG puede ser utilizado como depurador de ROS (principalmente superóxido y oxígeno singlete), 


\section{Table 1}

Concentración de ERG en cada proceso.

Producto/Proceso
Materia prima
EAH
EAH-UF(5KDa)
EAH-OI

$\begin{array}{ll}\text { ERG }(\mathbf{m g} / \mathbf{g}) & \text { ERG }(\mathbf{m g}) \\ 0,21 \pm 0,03 & 239,52 \\ 0,010 \pm 0,001 & 186,57 \\ 0,0090 \pm 0,0002 & 182,80 \\ 0,375 \pm 0,01 & 181,1 \\ 121,52 \pm 4,20 & 175,01\end{array}$

Los resultados se expresan como las medias \pm DE $(n=5)$ en peso seco.

esto podría atribuirse a la presencia de aminoácidos y péptidos libres que también podrían saciar las ROS.

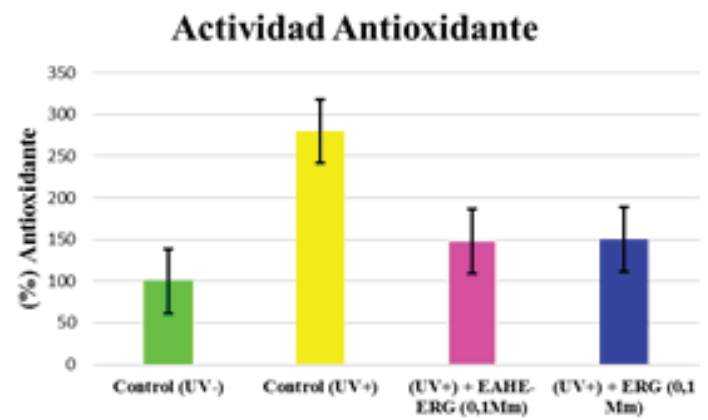

Figure 2

Porcentaje de antioxidante presente en cada muestra.

\subsection{Supresión de TNF- $\alpha$}

Los resultados de la Figura 3 muestran claramente que tanto EAHE-ERG como ERG pura, suprimen citocinas proinflamatorias como factor de necrosis tumoral $\alpha$ (TNF$\alpha$ ) e interleucina 12 (IL-12), las cuales activan la citotoxicidad celular dependiente de anticuerpos y activación de leucocitos inflamatorios, provocados por luz UV. Estos resultados indican que EAHE-ERG en concentraciones de 0,1 mM es suficiente para revertir procesos inflamatorios provocados por la exposicion a la luz ultravioleta, y así evitar el fotoenvejecimiento.

\subsection{Expresión MMP-1}

Las metaloproteinasas de matriz (MMP), desempeñan un papel importante en muchos procesos fisiológicos normales y también participan en muchos procesos patológicos como la artritis, el cáncer, las enfermedades cardiovasculares y la inflamación. Los resultados de la Figura 4 muestran que la luz UV induce la expresión de MMP-1 y que 


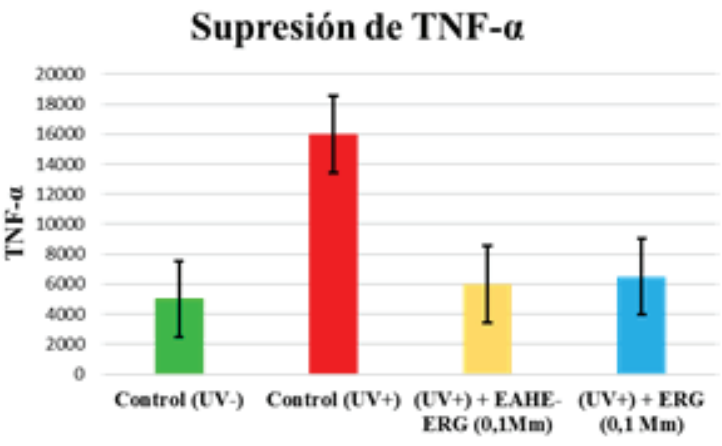

Figure 3

Nivel de TNF- $\alpha$ en las muestras.

EAHE-ERG a una concentración de 0,1 mM prácticamente redujo esta expresión en un $50 \%$ (resultados similares se obtienen mediante ERG pura, resultados no mostrados).

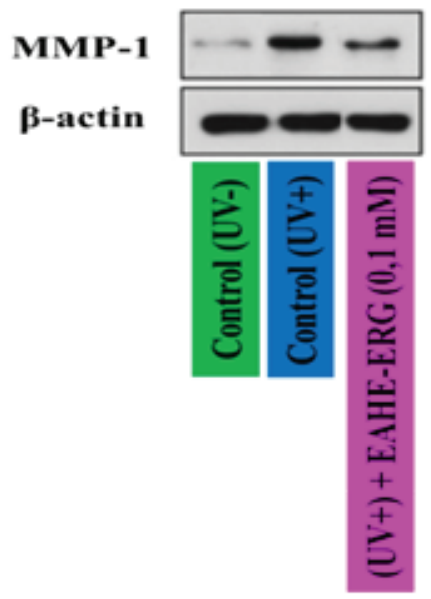

Figure 4

Expresión de MMP-1 en EAHE-ERG.

\section{Discusión}

Tang et al. fue el primero en estudiar la absorción y distribución de ERG en tejidos de ratones antes y después de la administración oral de ERG pura utilizando LC-MS/MS. Además, investigó la absorción y la farmacocinética de la L-ergotioneina después de la administración oral a humanos observando luego del tratamiento una tendencia decreciente de los biomarcadores de daño oxidativo y que sugiere que la ERG puede funcionar como un antioxidante importante, pero tal vez solo bajo condiciones de estrés oxidativo [19].

Muchos otros estudios in vitro han demostrado la capacidad de ERG para eliminar radicales, modular las respuestas inflamatorias y proteger contra la radiación UV y 
gamma [20, 21]. El objetivo de este estudio fue evaluar el efecto antioxidante de EAHEERG observándose que EAHE-ERG presenta un alto porcentaje antioxidante al igual que un extracto de ERG pura preparado a la misma concentración.

Las radiaciones UVA y UVB activan mecanismos inmunosupresores a través de ciertas citocinas, así contribuyen al daño y eventualmente a la carcinogénesis, los rayos UVA tienen un rol protagónico en la capacidad de penetrar los estratos más profundos de la piel, donde se producen los principales cambios del fotoenvejecimiento. La luz UV produce cambios moleculares provocando daño al tejido conectivo dérmico, causando la activación de receptores de factores de crecimiento, citocinas proinflamatorias (IL-1, TNF- $\alpha$, IL-6, IL-8) y moléculas de adhesión (ICAM-1) en la superficie de los queratinocitos y fibroblastos [22]. El TNF- $\alpha$ regula diversos eventos biológicos fundamentales de las células, como la activación de los leucocitos, la liberación de diversas citocinas y quimiocinas, y la producción de ROS e intermediarios de nitrógeno. El TNF- $\alpha$ es un agente pro-inflamatorio poderoso, liberado rápidamente después del trauma, infección o exposición a LPS y se ha demostrado que es el mediador más abundante en tejidos inflamados, es por eso que se ha tomado como un factor de estudio para comprobar el efecto que ejerce EAHE-ERG sobre los fibroblastos dérmicos humanos irradiados con UV. Nuestros resultados indican que EAHE-ERG suprime la regulación del incremento de TNF- $\alpha$ al igual que un extracto preparado con ERG pura, es decir que actúa como un antioxidante evitando la generación del ROS y por tanto evita los cambios moleculares y estructurales en la piel.

Las MMP, son enzimas secretadas por los fibroblastos y los queratinocitos, interfieren en la síntesis de degradación de los colágenos I y III dérmicos y de las proteínas de la matriz extracelular dérmica. La degradación del colágeno conduce a una acumulación de moléculas de colágeno parcialmente degradadas en la dermis, que alteran la integridad estructural de la piel. La luz UV induce la expresión de MMP-1, estimulando la producción de $\mathrm{H}_{2} \mathrm{O}_{2}$ provocando la inflamación o procesos patológicos como la artritis, el cáncer, las enfermedades cardiovasculares. EAHE-ERG a una concentración de 0,1 mM prácticamente redujo esta expresión en un 50\%. La MMP-1 o colagenasa intersticial, es capaz de iniciar la fragmentación del colágeno fibrilar tipo 1, que constituye $90 \%$ del colágeno de la piel [23].

\section{Conclusiones}

A partir de estos resultados, concluimos que EAHE-ERG, elimina el ROS generado por la fotosensibilización de tipo I y II y suprime tanto la expresión de TNF- $\alpha$ como la MMP-1 en su nivel de traducción. EAHE-ERG puede reducir los efectos antienvejecimiento de la piel después de la irradiación UV mediante la eliminación de $\mathrm{O}_{2}$ y $1 \mathrm{O}_{2}$, y la reducción de las señales de proteasa y actividad inflamatoria. En fibroblastos cultivados, EAHEERG suprimió la regulación por incremento de TNF- $\alpha$ mediante radiación UVB y cuando fueron expuestos a UVA, suprimió la expresión de MMP-1 en casi 50\%.

Debido a que EAHE-ERG posee la capacidad de interferir con la señalización molecular, ha llegado a ser un compuesto natural de gran interés por su potencial como agente terapéutico, y su posible uso como suplemento dietético. 


\section{Agradecimientos}

Al Ministerio de Ciencia español e Innovación para el apoyo financiero de este trabajo (Proyecto RTC2015-4039-2), que cuenta con apoyo parcial del FEDER. A la Universidad Técnica de Manabí por permitirnos colaborar con ellos.

\section{Conflicto de Intereses}

Declaro que no existen intereses particulares por parte de los autores o de la entidad científica que pudiesen afectar directa o indirectamente a los resultados.

\section{References}

[1] Melville DB. Ergothioneine. Vitamins and Hormones 1959;17:155-204.

[2] Halliwell B, Cheah IK, Tang RMY. Ergothioneine - A diet-derived antioxidant with therapeutic potential. FEBS Letters 2018;592:3357-3366.

[3] Park EJ, Lee WY, Kim ST, Ahn JK, Bae EK. Ergothioneine accumulation in a medicinal plant Gastrodia elata. Journal of Medicinal Plants Research 2010;4:1141-1147.

[4] Guo Q-L, Lin S, Wang YN, Zhu CG, Xu CB, Shi JG. Gastrolatathioneine, an unusual ergothioneine derivative from an aqueous extract of "tian ma": A natural product co-produced by plant and symbiotic fungus. Chinese Chemical Letters 2016;27:1577-1581.

[5] Cheah IK, Halliwell B. Ergothioneine; antioxidant potential, physiological function and role in disease. Biochimica et Biophysica Acta (BBA) - Molecular Basis of Disease 2012;1822(5):784-793.

[6] Tschirka J, Kreisor M, Betz J, Gründemann D. Substrate selectivity check of the ergothioneine transporter. Drug Metab. Dispos. 2018; 46: 779-785.

[7] Cheah IK, Tang RMY, Yew TSZ, Lim KHC, Halliwell B. Administration of pure ergothioneine to healthy human subjects: Uptake, metabolism, and effects on biomarkers of oxidative damage and inflammation. Antioxidants \& Redox Signaling 2017;26:193-206.

[8] Helfrich YR, Sachs DL, Voorhees JJ. Overview of skin aging and photoaging. Dermatology Nursing 2008;20(3):177.

[9] Ta P, Buchmeier N, Newton GL, Rawat M, Fahey RC. Organic hydroperoxide resistance protein and ergothioneine compensate for loss of mycothiol in Mycobacterium smegmatis mutants. Journal of Bacteriology 2011;193:1981-1990.

[10] Fisher GJ, Wang Z, Datta SC, Varani J, Kang S, Voorhees JJ. Pathophysiology of premature skin aging induced by ultraviolet light. The New England Journal of Medicine 1997;337(20):1419-1429.

[11] Yang NC, Lin HC, Wu JH, et al. Ergothioneine protects against neuronal injury induced by $\beta$-amyloid in mice. Food and Chemical Toxicology 2012;50:3902-3911.

[12] Sakrak Ö, Kerem M, Bedirli A, et al. Ergothioneine prevents acute lung injury in mesenteric ischemia and reperfusion injury in rats. Journal of Critical Care 2008;23:268-269.

[13] Fujitani Y, Alamgir KM, Tani A. Ergothioneine production using Methylobacterium species, yeast, and fungi. Journal of Bioscience and Bioengineering 2018;126:715-722.

[14] Takusagawa S, Satoh Y, Ohtsu I, Dairi T. Ergothioneine production with Aspergillus oryzae. Bioscience, Biotechnology, and Biochemistry 2019;83:181-184.

[15] Cremades O, Díaz-Herrero MM, Carbonero-Aguilar P, et al. White button mushroom ergothioneine aqueous extracts obtained by the application of enzymes and membrane technology. Food Bioscience 2015;10:42-47.

[16] Inca-Torres AR. Valorización de subproductos de la industria de hongos comestibles. Aplicación para obtener productos de alto valor agregado [Tesis Doctoral]. Sevilla, España: Universidad de Sevilla; 2019.

[17] Carbonero-Aguilar P, Gallego P, Falcón-García G, et al. Ergothioneine rich Agaricus Bisporus extracts decreases lipid accumulation induced by oleic acid in Hepg2 cells: Possible implications in the treatment of nonalcoholic liver fatty disease. Journal of Food Science and Nutrition 2019;5:049.

[18] Dubost NJ, Beelm RB, Peterson D, Royse DJ. Identification and quantification of ergothioneine in cultivated mushrooms using liquid chromatography mass spectroscopy. International Journal of Medicinal Mushrooms 2006;8:215-222. 
[19] Tang R, Cheah IK, Yew T, Halliwell B. Distribution and accumulation of dietary ergothioneine and its metabolites in mouse tissues. Scientific Reports 2018;8(1):1601.

[20] Motohashi N, Mori I, Sugiura Y, Tanaka H. Radioprotective effect of ergothioneine on gamma-irradiation of metmyoglobin: Comparison with cysteine on sulfmyoglobin-formation. Chemical and Pharmaceutical Bulletin 1977;25:2516-2523.

[21] Hartman PE, Hartman Z, Citardi MJ. Ergothioneine, histidine, and two naturally occurring histidine dipeptides as radioprotectors against gamma-irradiation inactivation of bacteriophages T4 and P22. Radiation Research 1988;114:319-330.

[22] Sánchez-Saldaña L, Lanchipa P, Pancorbo J, Regis A, Sánchez E. Fotoprotectores tópicos. Revista Peruana de Dermatología 2002;12(2).

[23] Morita A, Grewe M, Grether-Beck S, Olaizola-Horn S, Krutmann J. Induction of proinflammatory cytokines in human epidermoid carcinoma cells by in vitro ultraviolet A1 irradiation. Photochemistry and Photobiology 1997;65(4):630-635. 